Revista de Matemática: Teoría y Aplicaciones 2005 12(1 \& 2) : 165-171

CIMPA - UCR - CCSS ISSN: 1409-2433

\title{
PROGRAM IMPLEMENTATION OF THE RATING METHODS OF PREFERENCE RANKING
}

\author{
Kelly Torres* ${ }^{*}$ Alexey Sadovski ${ }^{\dagger} \quad$ Carl W. Steidley ${ }^{\ddagger}$
}

Received/Recibido: 13 Feb 2004

\begin{abstract}
Tide charts, based upon harmonic analysis, is the general method of choice for predicting water levels. In the shallow waters of the Gulf of Mexico, however, tide charts are woefully inadequate for the prediction of water levels. We have developed a number of models for the prediction of water levels. In this paper we summarize these methods and discuss the development of an axiomatic tool that we use to measure the quality of predictions of water levels in the estuaries and shallow waters of the Gulf of Mexico. This quality measure is based upon the preference rankings of National Ocean Service criteria by experts in the field.
\end{abstract}

Keywords: Rating methods, preference ranking, selection problem.

\section{Resumen}

Gráficos "tide", basados en análisis armónico, es el método general de escogencia para predecir niveles de agua. En aguas bajas del Golfo de México, sin embargo, gráficos "tide" no son adecuados para la predicción de niveles de agua. En este artículo resuminos estos métodos y discutimos el desarrollo de una herramienta axiomática que usamos para medir la calidad de las predicciones de niveles de agua en estuarios y aguas bajas del Golfo de México. Esta medida de calidad está basada en criterios de rankings de preferencia del Servicio Oceánico Nacional por expertos en el campo.

Palabras clave: Métodos de valoración, ranking de preferencias, problema de escogencia.

Mathematics Subject Classification: 90B50.

\footnotetext{
*Department of Computing and Mathematical Sciences, Texas A\& M University, 6300 Ocean Drive, Corpus Christi, TX, 78412 U.S.A.

${ }^{\dagger}$ Same address. E-Mail: sadovski@falcon.tamucc.edu.

${ }^{\ddagger}$ Same address. E-Mail: steidley@falcon.tamucc.edu.
} 


\section{Introduction}

Preference ranking is one of the methods to solve selection problems [1]. Selection problems are very important for decision making in unique systems such as medical, sports, environmental or ecological systems. Very often in such situations the right decision is based upon expert information. This paper presents theoretical results of the rating systems of preference ranking [2] as well as a practical realization of this approach in the form of the $\mathrm{C}++$ program. Theoretical results presented in this paper include the convergence of consensus ranking to the real ranking almost everywhere, and the inclusion of the consensus ranking into the Kemeny Median set [3]. It is shown also that all contemporary rating systems, (for instance those used in sports classifications), are congruent in the sense of producing the same final preference ranking. Such rating methods of preference ranking are very useful for solving multi-criteria problems of decision making and/or developing one goodness criteria from many different criteria [4].

\section{Preference ranking}

Let us consider a finite set of objects $A=\left\{a_{1}, \ldots, a_{n}\right\}$ and a set of $m$ experts $E$. Each expert presents a binary matrix of preferences $Q_{k}=\left(q_{i j}\right), k=1, \ldots, m$ and $i, j=1, \ldots, n$. The problem is to find a consensus ranking of objects based upon information provided by the experts. There are several ways to solve the selection problem under consideration. The first is to determine a Pareto set $P=\left\{P / \cap_{k} Q_{k} \subseteq P \subseteq Q_{k}\right\}$, but the set $P$ is too wide. The second method of the solution presented by Arrow [5] was based on a contradictory system of five axioms. The most useful result, obtained by Kemeny, is the so-called Kemeny Median $H=\left\{K / \sum d\left(K, Q_{k}\right)=\min \sum_{k} d\left(P, Q_{k}\right)\right\}$ which can be determined by methods of integer programming. It is necessary to outline, that the Kemeny Median satisfies four of the five Arrow axioms. There is also inclusion, i.e., $H \subseteq P$, but the set $H$ is still quite wide.

The methods presented in this paper provide advantages over other methods. First, there is a very simple numerical procedure. The second improvement is the possibility of using different forms of expert information such as preference ranking, allowing for binary and multi-comparison at the same time; this significantly differs from the methods based on uniform types of expert information. The third advantage is that the result of this rating procedure is a unique preference ranking and not just some set of suitable alternatives such as a Pareto Set or a Kemeny Median. Finally, some (or all) experts can present information of their preferences in the form of fuzzy relationships.

Let us consider this rating procedure more closely. Suppose there is some (maybe unknown to the decision-maker) order of objects $a_{1}, \ldots, a_{n}$ under consideration. Let us assume, that we have chosen some arbitrary scale, and each object has its own yet unknown value $r_{0}\left(a_{i}\right)$ in this chosen scale. The following is an axiom of existence:

Axiom 1 There is some order of given objects in any chosen scale.

Let us denote by $\Delta_{i j}=r_{0}\left(a_{i}\right)-r_{0}\left(a_{j}\right)$ the difference between real rating values. We believe that binary relationships given by experts satisfy 
Axiom 2 Fraction $\frac{\left[\text { number of preferences } a_{i} \succ a_{j}\right]}{\left[\text { number of preferences } a_{j} \succ a_{i}\right]}=f\left(\Delta_{i j}\right)$, where function $f(\Delta)$ is a positive strictly increasing function such that $f(0)=1$.

This assumption shows the odds or fuzzy odds of preferences by experts, who are asked to rank or compare objects.

Let $r_{i 0}, i=1, \ldots, n$ be some arbitrary initial ratings, where $r_{i k}$ is the rating of the $i$-th object after the $k$-th recalculation and $\Delta_{i j}(k)=r_{i}(k)-r_{j}(k)$. The following statement gives the simple way to calculate the ratings of objects according to the results of expert preferences.

\section{Axiom 3}

$$
r_{i}(k)=r_{i}(k-1)+q_{i j}(k) F\left(\Delta_{i j}(k-1)\right), r_{j}(k)=r_{j}(k-1)+q_{j i}(k) F\left(\Delta_{j i}(k-1)\right),
$$

where

$$
q_{i j}(k)= \begin{cases}1, & \text { if } a_{i} \succ a_{j} \\ 0, & \text { if } a_{i}=a_{i} \\ -1, & \text { if } a_{j} \succ a_{i}\end{cases}
$$

and $F(\Delta)$ is a nonnegative decreasing function.

In the case of fuzzy information, coefficients $q$ are equal values of a membership function of fuzzy binary relationships provided by experts respectively. For fuzzy relationships, the formulas in axiom 3 should be change as follows:

$$
\begin{aligned}
& r_{i}(k)=r_{i}(k-1)+q_{i j}(k) F\left(\Delta_{i j}(k-1)+q_{j i}(k) F\left(\Delta_{j i}(k-1)\right)\right. \\
& r_{j}(k)=r_{j}(k-1)+q_{j i}(k) F\left(\Delta_{j i}(k-1)+q_{i j}(k) F\left(\Delta_{i j}(k-1)\right) .\right.
\end{aligned}
$$

This means that an increase in rating value is proportional to a fuzzy preference and its decrease is proportional to a fuzzy non-preference.

It is reasonable that for a large $\Delta_{i j}$ the increase of $r_{i k}$ should be small, if $a_{i} \succ a_{j}$, but decreasing should be large if $a_{i} \prec a_{j}$. This idea is very useful, for instance, in sports methods of classifications: it means that if strong team or player ousts the weak one then there is almost no increase in the rating for the winner. However in the case of losing the game the higher rated team should loose many points. This is why we have two following assumptions for function $F$ :

Axiom $4 \lim _{\Delta \rightarrow \infty} F(\Delta)=0$.

Axiom $5 \lim _{\Delta \rightarrow-\infty} F(\Delta)=L>0$.

The following theorem establishes the equivalency of all rating systems of preference ranking including those rankings based on fuzzy information.

Theorem 1 For any initial ratings any method based on axioms 1 through 5 presents some preference ranking which is the same as a real unknown ranking with probability one in the space of realization, when $k \rightarrow \infty$. 
Theorem 2 The consensus preference ranking obtained as a result of rating procedure belongs, with probability one, to the Kemeny Median set in the space of all realizations $\Omega$.

It is easily seen that rating methods are iterative procedures for determining the Kemeny Median. The proof of this theorem is based on the fact that the Kemeny Median as well as a rating preference ranking satisfies four of the five Arrow axioms. Rating systems of preference ranking are very flexible. They provide an opportunity to work with different types of expert information such as binary and multi-comparison, ranking, etc. Moreover, it is possible to work with fuzzy information [6]. If, for instance, $\mu_{i j}$ is a measure of belonging that $a_{i} \succ a_{j}$ then it is enough to replace $q_{i j}$ by $\mu_{i j}$ in axiom 3 to use fuzzy relationship offered by experts. The last remark concerns the theorem of equivalency of rating systems, which also holds under conditions of fuzziness.

Competitive games, such as chess or tennis, use an integral estimator based on the results of a series of matches. Hence, this classification system is based on rankings. We have written a program for realization of such an approach using the $\mathrm{C}++$ language. Preference ranking is achieved by using a modified sports classification method developed by Professor A. Elo. Elo proposed a classification of chess players, which was adopted by the International Chess Federation in 1970. Arbitrary initial ratings are used. A group of decision-makers give their preferences based upon expert information. The experts compare two alternatives (binary comparison) or several alternatives (multi-comparison) and decide which alternative they prefer or if they are indifferent. Matrices of binary preferences or multi-preferences are then made to allow simpler comparison. The matrices are then entered into the program which implements the axiomatics of the rating system. The program computes the new ratings. Here we can use the method of tournaments, when the whole preference matrix is entered before recalculating new ratings, or we can use the method of matches, when new ratings are found after every comparison of two alternatives. There is no difference since the final consensus ranking must be the same. In our program, the method of matches was used. This approach can be used if there are multiple groups of decision-makers. After every expert's decision has been taken into account, a final standing is derived. Figure 1 shows the main steps of the program that were just mentioned above.

As mentioned above, the most well known rating system has been suggested by Elo, and it is used for the ranking of chess players. Elo postulated a 200 point difference between neighbor grades of players, and that the probability of winning by the more qualified player is equal to 0.75 . Further, he chose a scale and calculated ratings for all more or less well known players since the beginning of the 20th century. Using a simple function satisfying axiom 2, which is an exponent, we can determine the base of this function:

$$
\frac{a^{200}}{a^{200}}=0.75 \text { and } \alpha=1.0055
$$

The new rating in the Elo system is equal to old rating plus a ten fold difference between an actual result of the match and expected outcome of the game. 


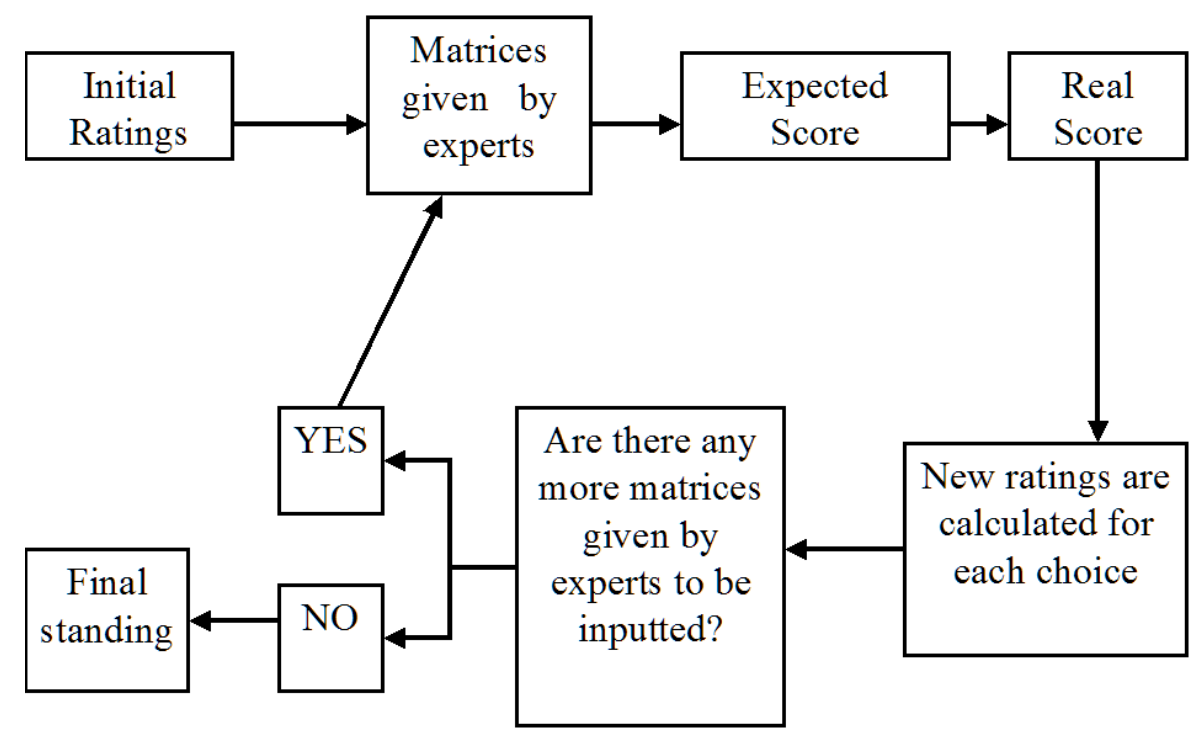

Figure 1: Overview of the ranking program.

\section{Ranking and Kemeny Median}

Assume that the experts present information about their preferences in matrix form. The Kemeny median is the set of such matrices where each element is the least distant from each other, and currently is the most useful consensus ranking, but to determine this median requires integer programming with all of its difficulties of calculation. It can be seen that rating methods are iterative procedures for determining the Kemeny Median. The ideal result for the ranking program is the converging of approximations of consensus ranking to the actual unknown consensus ranking and the inclusion of the consensus ranking into the Kemeny Median set. Assume that the experts present information about preferences in matrix form: $Q_{k}=\left(q_{i j}(k)\right), i, j=1, \ldots, n, k=1, \ldots, m$. For any two matrices of binary relations $Q_{k}$ and $Q_{l}$ the distance between them may be defined in the following way:

$$
d\left(Q_{k}, Q_{l}\right)=\frac{1}{2} \sum_{i=1}^{n} \sum_{j=1}^{n}\left[q_{i j}(k)-q_{i j}(l)\right] .
$$

If matrices $Q_{k}$ present preference ranking by experts then Kemeny median (4) is such matrix $K$ that

$$
\sum_{k=1}^{n} d\left(K, Q_{k}\right)=\min _{P} \sum_{k=1}^{n} d\left(P, Q_{k}\right) .
$$

The Kemeny median is actually a set of such matrices $K$, and at the present moment it is considered the most useful consensus ranking, again, however, the determination of this 
median requires integer programming with all its difficulties of calculation. The following result establishes the connection between the Kemeny median and rating rankings:

Theorem 3 The consensus preference ranking obtained as a result of rating procedure belongs, with probability one, to the Kemeny Median set in the space of all realizations $\Omega$.

\section{Ranking and Monte Carlo Method}

The Monte Carlo Method is a means of statistical evaluation of mathematical functions using random samples, and hence, requires a good source of random numbers. There is always some error involved with this method, but the larger the number of random samples taken, the more accurate the result. The Monte Carlo method is applied to the preference ranking program by randomly choosing which expert's preferences to apply first to the ranking. The application of the Monte Carlo method to this program helps to provide randomness for this implementation of system of preference ranking.

\section{Applications}

A preference ranking program has a wide range of applications. For instance, classification methods have been used for many years to rate athletes. Athletes are classified according to their strength, based on a number of points scored at tournaments or by a ranking system. It would be also beneficial in the medical field to know the important of criteria upon which to decide if certain drugs should be available to the public or to make a better diagnosis. Deciding upon "goodness" criteria for multi-objective decision making would also be made easier if we apply these rating methods of preference ranking. For example, we are using the program to rank experts' preferences for certain criteria from National Ocean Service skill assessment statistics used for evaluation of water level forecasts [4], [7], which are based on real-time data provided by Texas Coastal Ocean Observation Network.

As a result of such a procedure of preference ranking we can obtain ratings $r_{1}, \ldots, r_{n}$ for the given objectives. Suppose that we have the following ratings: $r_{1} \geq r_{2} \geq \ldots \geq r_{n}$. Let the value of coefficient $\alpha_{1}$ to be equal to one, in this case using the structure of the rating procedure we can find the remaining weight coefficients from the following relationship:

$$
\frac{\alpha_{1}}{\alpha_{i}}=f\left(\Delta_{1 i}\right), \quad i=1, \ldots, n,
$$

where $f$ and $\Delta_{i j}$ are defined by the described system of axioms mentioned above. These weight coefficients give us an opportunity to use the following additive integrated utility function:

$$
G=\alpha_{1} v_{1}(x)+\ldots+\alpha_{n} v_{n}(x)
$$

\section{Advantages}

The computational methods presented in this paper have several advantages. First, it is a very simple numerical procedure. The second advantage is the possibility of using different 
forms of expert information such as preference ranking, binary and multi-comparison at the same time; this significantly differs from other rating methods based on uniform types of expert information. The third advantage is that the result of this rating procedure provides a unique preference ranking and not just a set of suitable alternatives such as a Pareto Set or a Kemeny Median. Moreover, this unique consensus preference ranking belongs to Kemeny Median set. Similar results are obtained if some (or all) experts present information of their preferences in the form of fuzzy relationships.

\section{Acknowledgements}

The work presented in the paper is sponsored in part by National Aeronautics Space Agency (NASA grant \# NCC5-517) and Texas A\& M University-Corpus Christi.

\section{References}

[1] Arrow K.J. (1963) Social Choice and Individual Values. John Wiley\& Sons, New York.

[2] Sadovski A.L. (1989) "Application of expert methods in the decision making problems in conditions of fuzzy information", Voprosy Kibernetiky 151 (Russian), Academic Press, Moscow.

[3] Kemeny, J.; Snell, J. (1972) Mathematical Models in Social Sciences. The MIT Press, MA.

[4] Sadovski, A. L.; Steidley, C.; Tissot, P.; Michaud, P. (2003) "Developing a goodness criteria for tide predictions based on fuzzy preference ranking", in: Developments in Applied Artificial Intelligence, Lecture Notes in Artificial Intelligence 2718, Springer: 391-401.

[5] Sadovski, A.L. (2001) "Preference ranking and decisions based on fuzzy expert information", in: Advances in Fuzzy Systems and Evolutionary Computation, World Scientific Engineering Society Press, USA: 44-50.

[6] Sadovski, L.E.; Sadovski, A.L. (1993) Mathematics and Sports. American Mathematical Society, RI.

[7] NOS Procedures for Developing and Implementing Operational Nowcast and Forecast Systems for PORTS, National Oceanic and Atmospheric Administration, U.S. Department of Commerce, 1999.

[8] Michaud, P.; Jeffress, G.A.; Dannelly, R.S.; Steidley, C. (2001) "Real-time data collection and the Texas Coastal Ocean Observation Network", in: Proceedings of Emerging Technologies Conference, Instrument Society of America, Houston, Texas. 